A complete list of authors is available with the full text of this letter at NEJM.org.

Supported by a grant (HHSN272201400008C, to Dr. Kawaoka) from the Center for Research on Influenza Pathogenesis, funded by the National Institutes of Allergy and Infectious Diseases, and by a Research Program on Emerging and Re-emerging Infectious Disease grant (19fk0108113, to Dr. Kawaoka) from the Japan Agency for Medical Research and Development (AMED).

Disclosure forms provided by the authors are available with the full text of this letter at NEJM.org.

This letter was published on May 13, 2020, at NEJM.org.

1. 2 Cats in NY become the first US pets to test positive for virus. AP News. April 22, 2020 (https://apnews.com/ 37328ab8db093b8346e26e1840b48af8).
2. Shi J, Wen Z, Zhong G, et al. Susceptibility of ferrets, cats, dogs, and other domesticated animals to SARS-coronavirus 2. Science 2020 April 8 (Epub ahead of print).

3. Matsuyama S, Nao N, Shirato K, et al. Enhanced isolation of SARS-CoV-2 by TMPRSS2-expressing cells. Proc Natl Acad Sci U S A 2020;117:7001-3.

4. United States Department of Agriculture, Animal and Plant Health Inspection Service. USDA statement on the confirmation of COVID-19 in a tiger in New York. April 6, 2020 (https://www .aphis.usda.gov/aphis/newsroom/news/sa_by_date/sa-2020/ny-zoo -covid-19).

5. Marinova-Petkova A, Laplante J, Jang Y, et al. Avian influenza A(H7N2) virus in human exposed to sick cats, New York, USA, 2016. Emerg Infect Dis 2017;23:2046-9.

DOI: 10.1056/NEJMc2013400

\title{
Five-Year Outcomes with Transcatheter Aortic-Valve Replacement
}

TO THE EDITOR: The key message of the report by Makkar et al. ${ }^{1}$ and the editorial by Van Belle ${ }^{2}$ (Feb. 27 issue) on the Placement of Aortic Transcatheter Valves (PARTNER) 2 cohort A trial is the effectiveness of transcatheter aortic-valve replacement (TAVR) in terms of death from any cause or disabling stroke at 5 years. Nonetheless, on critical appraisal, other important results are noted. The landmark analysis of events occurring between 2 and 5 years after the procedure (Fig. S4 in the Supplementary Appendix of the article, available at NEJM.org) shows that TAVR was associated with a higher risk of death or disabling stroke after 2 years than was surgical aortic-valve replacement, with a hazard that was $27 \%$ higher. Moreover, the intersection of the time-to-event curves (Fig. 1 of the article) and the wide differences between the hazard ratio at 0 to 2 years (0.89; $95 \%$ confidence interval [CI], 0.73 to 1.09 ) and that at 2 to 5 years $(1.27 ; 95 \%$ CI, 1.06 to 1.53) suggest that the hazards were not constant over time. Therefore, the reported 5-year hazard ratio is not an accurate reflection of the findings, which require landmark analysis or time-varying modeling. ${ }^{3,4}$ The key message of the trial is also overshadowed by the disadvantage of TAVR in terms of reoperations and rehospitalization, which underscores concerns about the durability of TAVR devices. In summary, the 5-year results from the PARTNER 2 cohort A trial are not a swan song for surgery.

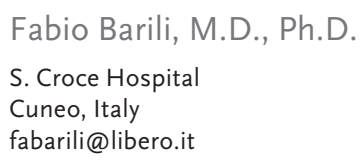

\section{Nicholas Freemantle, M.D.}

University College London

London, United Kingdom

Alessandro Parolari, M.D., Ph.D.

IRCCS Policlinico S. Donato

Milan, Italy

Dr. Barili reports receiving fees from Abbott Medical for serving on an independent clinical event committee for a trial of the Trifecta valve; and Dr. Freemantle, receiving grants from the European Association for Cardio-Thoracic Surgery. No other potential conflict of interest relevant to this letter was reported.

1. Makkar RR, Thourani VH, Mack MJ, et al. Five-year outcomes of transcatheter or surgical aortic-valve replacement. $\mathrm{N}$ Engl J Med 2020;382:799-809.

2. Van Belle E. TAVR at 5 years - rematch or swan song for surgery? N Engl J Med 2020;382:867-8.

3. Putter $\mathrm{H}$, van Houwelingen HC. Understanding landmarking and its relation with time-dependent Cox regression. Stat Biosci 2017;9:489-503.

4. Barili F, Freemantle N, Pilozzi Casado A, et al. Mortality in trials on transcatheter aortic valve implantation versus surgical aortic valve replacement: a pooled meta-analysis of Kaplan-Meierderived individual patient data. Eur J Cardiothorac Surg 2020 April 1 (Epub ahead of print).

DOI: 10.1056/NEJMc2018853

TO THE EDITOR: The report by Makkar et al. from the PARTNER 2 cohort A trial shows that TAVR is similar to surgery in terms of death and disabling stroke at 5 years. However, TAVR resulted in a smaller reduction of both left ventricular end diastolic volume and left ventricular mass index (Table 1). These differences in left ventricular regression occurred within 30 days and persisted up to 5 years after implantation; they have been replicated elsewhere. ${ }^{1}$

Reduced left ventricular regression has previously been associated with increased rehospital- 
izations and heart-failure admissions, ${ }^{2}$ both of which were observed in the TAVR cohort of this trial (Table S9). No clear explanation exists as to why TAVR results in less left ventricular regression than surgery, with increased paravalvular leak or pacemaker implantation found previously not to have an effect on left ventricular regression. ${ }^{1}$ With TAVR expanding toward patients with intermediate or even lower risk, attention should now focus on identifying potential clinical, procedural, or device-related factors that affect left ventricular regression in order to improve clinical outcomes.

Arif A. Khokhar, B.M., B.Ch.

Francesco Giannini, M.D.

Antonio Colombo, M.D.

Maria Cecilia Hospital

Cotignola, Italy

arifkhokhar@doctors.org.uk

No potential conflict of interest relevant to this letter was reported.

1. Ngo A, Hassager C, Thyregod HGH, et al. Differences in left ventricular remodelling in patients with aortic stenosis treated with transcatheter aortic valve replacement with corevalve prostheses compared to surgery with porcine or bovine biological prostheses. Eur Heart J Cardiovasc Imaging 2018;19:39-46.

2. Lindman BR, Stewart WJ, Pibarot P, et al. Early regression of severe left ventricular hypertrophy after transcatheter aortic valve replacement is associated with decreased hospitalizations. JACC Cardiovasc Interv 2014;7:662-73.

DOI: $10.1056 /$ NEJMc2018853

THE AUTHORS REPLY: We agree with Barili et al. that the higher risk of death or disabling stroke between 2 and 5 years after TAVR than after surgery in the landmark analysis arouses concerns regarding the long-term effectiveness of TAVR. Although there were wide differences between hazard ratios at 0 to 2 years and at 2 to 5 years, the hazard ratios that were calculated for later time periods include only survivors of earlier time periods and thus are not truly randomized comparisons. Furthermore, a similar 5-year restricted mean survival time (event-free time for the primary end point) in the TAVR and surgery groups supports the validity of similar effectiveness with respect to death or disabling stroke at 5 years. It is important to emphasize that these results were obtained with a previous-generation valve limited by higher rates of paravalvular regurgitation than newer-generation valves and $25 \%$ usage of the transthoracic approach that has now largely been abandoned owing to worse outcomes. The comparisons between TAVR and sur-

\begin{tabular}{|lcc|}
$\begin{array}{l}\text { Table } 1 . \text { Changes in Indexes of Left Ventricular Regression after TAVR } \\
\text { as Compared with Surgery.* }\end{array}$ & \multicolumn{1}{l|}{ Surgery } \\
\hline Variable & TAVR & \\
Left ventricular end-diastolic volume $(\mathrm{ml})$ & & \\
30-Day change from baseline & $0.55 \pm 20.10$ & $-11.01 \pm 20.40$ \\
2-Yr change from baseline & $-3.01 \pm 27.38$ & $-16.19 \pm 28.87$ \\
5-Yr change from baseline & $-4.03 \pm 31.60$ & $-20.54 \pm 32.81$ \\
Left ventricular mass index $\left(\mathrm{g} / \mathrm{m}^{2}\right)$ & & \\
30-Day change from baseline & $-3.74 \pm 20.39$ & $-13.46 \pm 22.91$ \\
2-Yr change from baseline & $-10.06 \pm 24.06$ & $-18.16 \pm 25.07$ \\
5-Yr change from baseline & $-13.89 \pm 26.56$ & $-22.59 \pm 26.91$ \\
\end{tabular}

* The data are from Table S12 in the Supplementary Appendix of the article by Makkar et al. Plus-minus values are means \pm SD.

gery in the transfemoral cohort are more representative of current practice (Fig. S2A in the Supplementary Appendix of our article).

Khokhar et al. point to the more favorable reductions in left ventricular volumes and mass regression in patients treated with surgery as compared with TAVR. Postprocedure moderate or severe aortic regurgitation has been independently associated with less left ventricular mass regression in TAVR. ${ }^{1}$ In the current data, the differences in left ventricular mass regression between TAVR and surgery persisted after excluding patients with at least moderate aortic regurgitation. The effect of additional procedural outcomes including new pacemaker and conduction-system block on left ventricular regression needs further elucidation. A recent analysis of the PARTNER 3 trial $^{2}$ involving low-risk patients showed equivalent left ventricular mass regression both at 30 days and 1 year for TAVR and surgical aortic-valve replacement, which suggests that the hemodynamics of the SAPIEN 3 valve may have advantages over the SAPIEN XT valve used in our trial.

The results of the recent trials showing superiority or noninferiority of TAVR over surgery in low-risk patients at 1 and 2 years ${ }^{3,4}$ and noninferiority to surgery in intermediate-risk patients at 5 years justify careful expansion of TAVR independent of patients' risk of surgery. Nonetheless, given the lack of long-term data, our trial is not a swan song for surgery.

Raj R. Makkar, M.D.

Cedars-Sinai Medical Center

Los Angeles, CA

raj.makkar@cshs.org 
Michael J. Mack, M.D.

Baylor Scott and White Health

Plano, TX

Martin B. Leon, M.D.

Columbia University Irving Medical Center

New York, NY

Since publication of their article, the authors report no further potential conflict of interest.

1. Chau KH, Douglas PS, Pibarot P, et al. Regression of left ventricular mass after transcatheter aortic valve replacement: the PARTNER trials and registries. J Am Coll Cardiol 2020;75: 2446-58.

2. Pibarot P, Salaun E, Dahou A, et al. Echocardiographic results of transcatheter versus surgical aortic valve replacement in low-risk patients: the PARTNER 3 trial. Circulation 2020;141: 1527-37.

3. Mack MJ, Leon MB, Thourani VH, et al. Transcatheter aorticvalve replacement with a balloon-expandable valve in low-risk patients. N Engl J Med 2019;380:1695-705.

4. Popma JJ, Deeb GM, Yakubov SJ, et al. Transcatheter aortic valve replacement with a self-expanding valve in low-risk patients. N Engl J Med 2019;380:1706-15.

DOI: 10.1056/NEJMc2018853

THE EDITORIALIST AND COLLEAGUES REPLY: We thank Barili et al. for their methodologic point regarding the trial by Makkar et al. reporting the 5 -year outcomes of the comparison of surgical aortic-valve replacement versus TAVR with the SAPIEN XT valve (PARTNER 2 cohort A trial). They noted that the proportional-hazards assumption was not verified over time, as easily spotted by the crossing of the Kaplan-Meier curves after 2 years of follow-up. Makkar et al. anticipated this possibility and prespecified the calculation of the restricted mean survival time and the restricted mean event-free time for the primary end point (death or disabling stroke). ${ }^{1}$ These results, which are reported in Table S8 and in the text, show that the 5-year restricted mean survival time was similar for TAVR and surgery (46.3 months and 46.6 months, respectively), as was the restricted mean event-free time for the end point of death or disabling stroke (45.0 months and 44.8 months). They also performed a landmark analysis showing superiority of surgery beyond 2 years (hazard ratio with TAVR, 1.27; 95\% CI, 1.06 to 1.53) (Table S7). The potential explanations for this late increase in risk with TAVR were extensively discussed in the editorial, including the potential role of paravalvular regurgitation, the lack of concomitant coronary or valve procedures, and durability concerns.

Khokhar et al. commented that the magnitude of the left ventricular mass regression was less pronounced in the TAVR group than in the surgery group. We can reasonably speculate that the higher rate of mild or greater paravalvular regurgitation with the second-generation transcatheter bioprosthesis than with the surgical valve may have adversely influenced left ventricle remodeling. ${ }^{2}$ The recently published echocardiographic outcomes of low-risk patients in the PARTNER 3 trial are supportive of this hypothesis, since the very low rate of aortic regurgitation that was achieved with the third-generation valve (SAPIEN 3) was associated with left ventricular mass regression similar to that with surgery at 1 year $(-11.9 \pm 21.10$ vs. $-12.0 \pm 27.09 \mathrm{~g}$ per square meter, $\mathrm{P}=0.84)$. $^{3}$

Eric Van Belle, M.D., Ph.D.

Institut Cœur Poumon

Lille, France

Julien Labreuche, B.S.T.

Université de Lille

Lille, France

Flavien Vincent, M.D., Ph.D.

Centre Hospitalier Universitaire de Lille Lille, France

Mr. Labreuche and Dr. Vincent report no potential conflict of interest. Since publication of his editorial, Dr. Van Belle reports no further potential conflict of interest.

1. Royston P, Parmar MKB. Restricted mean survival time: an alternative to the hazard ratio for the design and analysis of randomized trials with a time-to-event outcome. BMC Med Res Methodol 2013;13:152.

2. Van Belle E, Vincent F, Labreuche J, et al. Balloon-expandable versus self-expanding transcatheter aortic valve replacement: a propensity-matched comparison from the FRANCE-TAVI registry. Circulation 2020;141:243-59.

3. Pibarot P, Salaun E, Dahou A, et al. Echocardiographic results of transcatheter versus surgical aortic valve replacement in low-risk patients: the PARTNER 3 trial. Circulation 2020;141: 1527-37.

DOI: 10.1056/NEJMc2018853

\section{Emapalumab in Primary Hemophagocytic Lymphohistiocytosis}

TO THE EDITOR: New and affordable treatments for primary hemophagocytic lymphohistiocytosis (pHLH) are valuable. However, the study by Locatelli et al. (May 7 issue) ${ }^{1}$ does not present con- vincing data for strong efficacy of emapalumab in patients with pHLH. The data reveal that among a total of 34 patients, including 27 who had previously received treatment and 7 who had 